\title{
Ischemia-modified albumin in migraine patients during interictal period
}

\author{
Sławomir Michalak', Krystyna Osztynowicz' Alicja Płóciniczak², Waldemar Myszka², \\ Marcin Nowicki ${ }^{2}$, Danuta Węgrzyn ${ }^{3}$, Małgorzata Drzewiecka ${ }^{3}$, Kinga Kapecka ${ }^{4}$, \\ Wojciech Kozubski ${ }^{3}$ \\ 1 Department of Neurochemistry and Neuropathology, Poznan University of Medical Sciences, Poland \\ ${ }^{2}$ Department of Clinical Biochemistry and Laboratory Medicine, Poznan University of Medical Sciences, Poland \\ ${ }^{3}$ Department of Neurology, Poznan University of Medical Sciences, Poland \\ ${ }^{4}$ Reological Laboratory, Department of Neurology, Poznan University of Medical Sciences, Poland
}

\begin{abstract}
Aim. Ischemia-modified albumin (IMA) is a marker of myocardial ischemia and may be affected by ischemia occurring in other tissues. Migraine has been reported as a risk factor of ischemic stroke or cardiovascular events. Dysfunction of endothelial cells, as well as association with arteriopathies was evidenced in migraine patients. The aim of this study was to evaluate interictal IMA in migraine patients.

Material and Methods. Fifty migraineurs aged $38 \pm 9$ years were included in the study. The control group consisted of 25 healthy volunteers aged $37 \pm 8$ years. In all subjects neurological examination was carried on, as well as clinimetric evaluation with the use of: MIDAS, MIGSEV, QVM, VAS and VRS. Ischemia-modified albumin was evaluated by means of spectrophotometric method with the use of cobalt chloride. The concentrations of total cholesterol, HDL- and LDL-cholesterol, triglycerides, homocysteine, C-reactive protein and Lp(a) were analyzed with routine spectrophotometric methods.

Results. IMA was significantly $(P=0.0108)$ higher in migraine patients $(0.101 ; 0.00-0.327$ O.D.) than in controls (0.00; 0.00-0.102 O.D.; median; interquartile range). Migraineurs with aura have also higher IMA than controls. IMA correlated $(r S=0.383, P=0.0073$ ) with VAS and with homocysteine concentration $(r S=0.430, P=0.0026)$. Multiple regression analysis of IMA and atherosclerosis risk factors showed significant correlation $(P=0.0247)$ with HDL cholesterol $(R=0.2958)$ and triglycerides concentrations $(R=0.3285)$.

Conclusions. IMA formation in migraine patients, as a marker of oxidative stress even during interictal period in patients with hyperhomocysteinemia and/or hypertriacylglyceridemia can reflect a milieu of factors which further increases the risk for cardiovascular complications.
\end{abstract}

Keywords: headache, ischemia-modified albumin, oxidative stress, cerebrovascular diseases.

\section{Introduction}

The pathomechanisms considered in migraine involve complex milieu of factors acting both on neural and vascular compartments in the brain. "Stroke-migraine continuum" hypothesis links both sides of those pathomechanisms [1]. Depolarization which propagates across grey matter in the brain, known as cortical spreading depression (CSD) or spreading depolar- ization (SD), is related to neuronal compartment and contributes to the development of aura symptoms [2, 3]. The relations between CSD and cerebral blood flow (CBF) show quadriphasic pattern: short CBF decrease marked CBF increase - minor, but sustained CBF elevation - sustained CBF decrease (oligemia) [4]. Oligemia related to CSD, constriction of intracerebral large arter- 
ies and endothelium-related hypercoagulability may cause cerebral ischemia in migraine patients [5].

Migraine is considered not only as a risk factor of stroke, but also of other cardiovascular diseases. Meta-analysis showed that the risk of ischemic stroke in migraine patients is more than 2 times higher compared to nonmigraineurs [6], but the results for hemorrhagic stroke are inconsistent [7]. In the HUNT Study (Nord-Trøndelag Health Study in Norway) [8] Framingham risk score, which includes age, sex, total cholesterol and HDL-cholesterol concentrations, smoking status and systolic blood pressure stratified for the use of antihypertensive medication was used as indicator of cardiovascular risk in headache patients [9]. In non-migraine headache patients, migraineurs without aura and migraineurs with aura Framingham risk score was increased compared to controls [8]. The study done in USA also showed that migraine, both with and without aura, is associated with higher risk for cardiovascular diseases including myocardial infarction, stroke, and claudication [10].

Human serum albumin is the main plasma protein. It consists of 585 amino acids formed into a single polypeptide with three homologous helical domains. First three amino acids in $\mathrm{N}$ - terminal region form specific binding sites for transition metals like cobalt, copper, nickel and are the most susceptible region for degradation compared to other regions of albumin. The mechanisms involved in ischemia/reperfusion, especially those related to oxidative stress, cause changes to albumin and reduce the transitional metal binding capacity [11]. Such a transformed albumin is called ischemia-modified albumin (IMA). It is proposed to be a biomarker of ischemic heart disease, as it has been noticed that serum albumin of individuals with myocardial ischemia exhibits reduced biding to cobalt in comparison to non-ischemic ones [11]. What is more, this reduced biding of cobalt (Co(II)) to serum albumin was also observed among patients with transient myocardial ischemia after elective percutaneous transluminal coronary angioplasty [12]. IMA was increased in cases of oxidative stress due to ischemia reperfusion injury $[13,14,15]$, thus may distinguish between ischemic and non-ischemic patients, which may be clinically important as far as immediate diagnose is concerned.

With this background in mind we have undertaken the study on IMA in migraine patients during interictal period. The aim of the study was to evaluate IMA in migraineurs with and without aura and to correlate it with cardiovascular risk factors determined.

\section{Material and Methods}

\section{Patients}

We have included in the study 50 migraine patients aged $38 \pm 9$ and diagnosed basing on the criteria of International Headache Society. The exclusion criteria were: history of cardiovascular disease, hypertension (defined as systolic blood pressure exceeding $140 \mathrm{~mm}$ $\mathrm{Hg}$ or diastolic blood pressure over $90 \mathrm{~mm} \mathrm{Hg}$ ), diabetes, hyperlipidemia, pregnancy or lactation, inflammation, allergy, and regular use of vasoactive drugs (except hormonal contraceptives).

The control group consisted of 25 healthy volunteers aged $37 \pm 8$ years.

None of migraine patients and controls showed symptoms of any acute or chronic disease in medical history, during physical examination, and in routine laboratory tests.

All study participants underwent neurological examination and clinimetric evaluation with the use of: MIGSEV (4-item questionnaire which evaluates migraine intensity as mild, moderate or severe basing on pain, nausea, limitation of everyday activity and tolerability of symptoms) [16], MIDAS (Migraine Disability Assessment measures migraine - associated disability basing on 7 questions) [17], QVM (Qualité de Vie et Migraine evaluates quality of life basing on 20 questions) [18], VAS (Visual Analogue Scale: 0 to 10 points, with 10 indicating the most severe pain) and VRS (four-point verbal rating scale: 0 to 3 , with 3 for severe pain).

Fasting blood samples were collected in the morning not earlier than 4 days after migraine attack and/ or administration of triptans or ergot alkaloids. Routine laboratory tests were performed immediately after blood collection. Samples for IMA evaluation were stored at $-80^{\circ} \mathrm{C}$ until analysis. White blood cells count (WBC) was evaluated with the use of automated hematology analyzer. C-reactive protein (CRP), total cholesterol, high-density lipoprotein cholesterol (HDL), low-density lipoprotein cholesterol (LDL), triglycerides (TAG) concentrations were analysed with routine methods. Lipoprotein a $(L p(a))$ and homocystein concentrations were analyzed by means of enzyme-linked immunosorbent assay (ELISA).

\section{Measurements}

Ischemia modified albumin was estimated spectrophotometrically using Sigma-Aldrich (Germany) reagents and spectrophotometer Statfax 1904 Plus (Awareness Technology, USA), according to the manual method developed by Bar-Or et al $[19,20]$. 
The assay is based on the statement that ischemia (first reported for myocardial ischemia) causes some changes in human plasma albumin resulting in reduced binding of exogenous cobalt, $\mathrm{Co}(\mathrm{II})$.

The unbound exogenous cobalt (Co) reacts with colored marker (dithiothreitol, DTT) and increased intensity of the reaction reflects a reduced metal binding capacity of albumin, thus increased modification of albumin. The result of the determination is expressed as optical density (O.D.) [20, 21].

\section{The assay procedure}

Patients serum $(200 \mu \mathrm{l})$ was added to $50 \mu \mathrm{l}$ of a cobalt chloride solution $(1 \mathrm{~g} / \mathrm{l})$. Next, vigorous mixing and a 10-minute incubation in standard room temperature were performed. After then, $50 \mu \mathrm{l}$ of $1.5 \mathrm{~g} / \mathrm{I}$ DTT solution was added, what was followed by mixing and 2-minute incubation in standard room temperature. Finally, $1.0 \mathrm{ml}$ of $9.0 \mathrm{~g} / \mathrm{l}$ solution of $\mathrm{NaCl}$ was added to obtain the assay sample. The procedure of preparing the blank sample required $50 \mu$ of double-distilled water instead of DTT. The absorbance of the assay sample was read at 470 $\mathrm{nm}$ against the blank sample, in duplicate.

The intra- and inter-assay coefficients of variation were $4,8 \%$ and $6,2 \%$, respectively.

\section{Statistics}

Statistical analysis was performed with the use of a licensed version of MedCalc 16.8.4 (64 bit) software. First, the distribution of results was analyzed with the D'Agostino-Pearson test. The variables that had a normal distribution were expressed as mean \pm SD and tested with t-Student test; the variables that had a non-gaussian distribution were expressed as median and interquartile range and analyzed with the Mann-Whitney test.
Ethics

All participants signed written informed consent. The study protocol was approved by the Ethic Committee at the Poznan University of Medical Sciences.

\section{Results}

Neurological examination of migraine patients did not revealed any deficits. With the use of clinimetric measures we have noticed high intensity of pain reflected by VAS and VRS scores, intermediate to high severity of attacks in MIGSEV score, and severe functional impairment in MIDAS and QVM scale (Table 1). There were no differences in clinimetric scores between migraineurs with and without aura (Table 1).

Total cholesterol concentrations were higher in migraine patients with and without aura compared to controls. In migraineurs with aura HDL cholesterol levels were higher than in controls. We did not observed differences in results of other routine laboratory tests between migraine subjects and controls (Table 2 ).

IMA was significantly $(P=0.0108)$ higher in the whole group of migraine patients $(0.101 ; 0.00$ to $0.3270 . \mathrm{D}$.) than in controls $(0.00 ; 0.00$ to 0.102 0.D.; median; interquartile range). The subgroup of migraineurs with aura have also higher IMA than controls (Table 3 ).

IMA correlated $(r S=0.383, P=0.0073)$ significantly with clinimetric evaluation with the use of VAS (Figure 1). Homocysteine was the only result in the panel of routine laboratory tests which correlated with IMA $(r S=0.430, P=0.0026)$ (Figure 2).

In multiple regression analysis of relation between IMA and plasma lipids in the model including total cholesterol, HDL cholesterol, LDL cholesterol, triglycerides (TAG) and $L p(a)$ concentrations we have found

Table 1. The severity of migraine symptoms in migraine patients with and without aura

\begin{tabular}{lccc}
\hline & $\begin{array}{c}\text { Migraine with aura } \\
\mathrm{N}=23\end{array}$ & $\begin{array}{c}\text { Migraine without aura } \\
\mathrm{N}=27\end{array}$ & $\mathrm{p}$ \\
\hline VAS (Visual Analogue scale) & $7.96 \pm 1.64$ & $8.24 \pm 1.33$ & $\mathrm{NS}$ \\
\hline $\begin{array}{l}\text { VRS } \\
\text { (four - point Verbal Rating Scale) }\end{array}$ & $2.65 \pm 0.57$ & $2.88 \pm 0.33$ & $\mathrm{NS}$ \\
\hline QVM Global Index & $26.17 \pm 6.68$ & $27.20 \pm 5.48$ & $\mathrm{NS}$ \\
\hline MIDAS & $34.48 \pm 16.50$ & $42.16 \pm 18.87$ & $\mathrm{NS}$ \\
\hline MIGSEV - Pain & 4.0 & 3.0 & \\
\hline MIGSEV- Nausea & $2.0-4.0$ & $3.0-4.0$ & $\mathrm{NS}$ \\
\hline MIGSEV- Disability in daily activity & $3.3 \pm 0.7$ & $3.3 \pm 0.5$ & $\mathrm{NS}$ \\
\hline MIGSEV- Tolerability & $3.04 \pm 0.82$ & $3.20 \pm 0.50$ & $\mathrm{NS}$ \\
\hline
\end{tabular}

Variables with gaussian distribution are presented as mean $\pm S D$, variables with non-gaussian distribution as median; interquartile range 
Table 2. Results of routine laboratory tests in migraineurs and controls

\begin{tabular}{lccc}
\hline & Controls & Migraine with aura & Migraine without aura \\
\hline \multirow{2}{*}{ WBC } & 6.1 & 5.8 & 5.2 \\
& $4.9-7.8$ & $4.1-7.2$ & $4.7-7.1$ \\
\hline CRP & 0.64 & 0.46 & 0.42 \\
& $0.16-1.02$ & $0.17-0.82$ & $0.24-1.10$ \\
\hline Glucose [mg/dL] & 71 & 66 & 81 \\
\hline Total cholesterol [mg/ & $62-83$ & $49-68$ & $70-90$ \\
\hline dL] & $184 \pm 35$ & $208 \pm 36^{*}$ & $227 \pm 40^{+}$ \\
\hline HDL cholesterol [mg/ & $59 \pm 12,6045$ & $67 \pm 12^{\#}$ & $66 \pm 14$ \\
dL] & $114 \pm 44$ & $126 \pm 40$ & $141 \pm 34^{\S}$ \\
\hline LDL cholesterol [mg/dL] & 77 & 90 & 98 \\
\hline TAG [mg/dL] & $55-155$ & $64-118$ & $67-126$ \\
\hline Lp (a) [g/L] & 0.08 & 0.09 & 0.06 \\
\hline Homocysteine [mmol/L] & 12.12 & 12.25 & $0.032-0.285$ \\
\hline \multirow{2}{*}{ Albumin [g/L] } & $9.24-13.33$ & $10.58-14.38$ & 12.20 \\
& 61.7 & 62.0 & $63-15.14$ \\
\hline
\end{tabular}

Variables with gaussian distribution are presented as mean $\pm S D$, variables with non-gaussian distribution as median; interquartile range

* $-P=0,0243 ;+-P=0,0002$ - compared to controls; \#-P=0,0287 - compared to controls; $\$-P=0,0190$ - compared to controls

Table 3. IMA in migraine patients and controls

\begin{tabular}{|c|c|c|c|c|}
\hline & Controls & Migraineurs total & Migraine with aura & Migraine without aura \\
\hline $\begin{array}{l}\text { IMA } \\
\text { [0.D.] } \\
\text { median } \\
\text { interquartile range }\end{array}$ & $\begin{array}{c}0.0 \\
0.0-0.102\end{array}$ & $\begin{array}{c}0.101^{*} \\
0.0-0.327\end{array}$ & $\begin{array}{c}0.073^{+} \\
0.014-0.346\end{array}$ & $\begin{array}{c}0.111 \\
0.0-0.235\end{array}$ \\
\hline $\begin{array}{l}\text { IMA } \\
\text { [0.D.] } \\
\text { minimum - } \\
\text { maximum }\end{array}$ & $0.0-0.511$ & $0.0-1.932$ & $0.0-1.922$ & $0.0-1.932$ \\
\hline
\end{tabular}

*- $P=0.0108 ;+-P=0.0103$

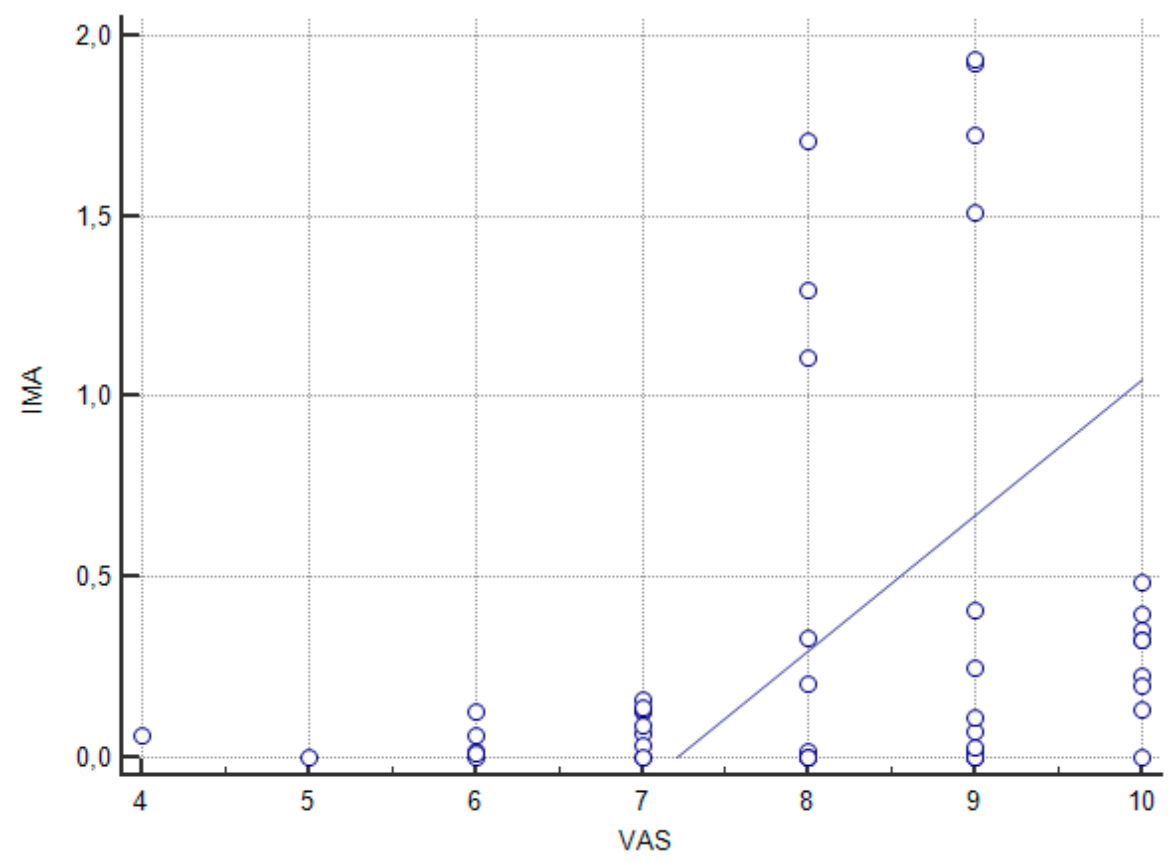

Figure 1. Correlation between IMA and VAS in migraine patients 


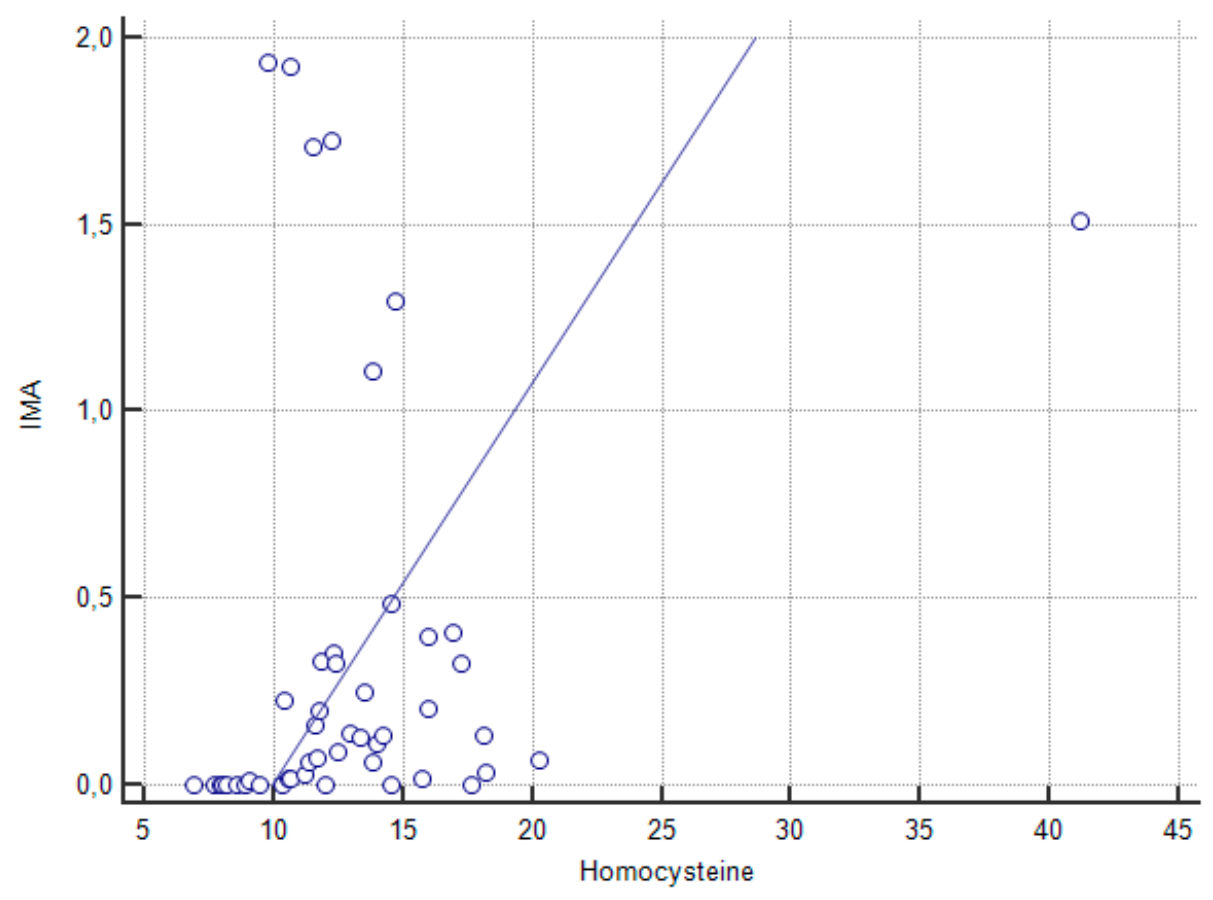

Figure 2. Correlation between IMA and homocysteine concentration in migraine patients

that IMA correlates $(P=0.0247)$ with $\mathrm{HDL}$ cholesterol $(R=0.2958)$ and $T A G$ concentrations $(R=0.3285)$.

\section{Discussion}

We report the stimulation of ischemia-modified albumin formation in migraine patients during interictal period. In our study IMA correlated both with pain intensity, pro-atherosclerotic risk factors (homocysteine, triglycerides) and with anti-atherosclerotic marker (HDL cholesterol). We are not aware of the studies on IMA in migraine patients, thus it is an initial report on this topic.

IMA as a novel biomarker of oxidative stress and together with low grade systemic inflammation are underlying causes of many diseases. IMA plays a major role in their prediction before the overt onset. It has been reported that IMA is elevated in ischemic heart disease and as it increases within first minutes after ischemia and thus it was considered as the earliest predictor of myocardial infarction [12].

When ischemic changes started with hypoxia, free oxygen radicals lead to peripheral vascular insufficiency, which resulted in ischemia of the limbs and ischemic heart disease. Gunduz et al [22] noticed that there was a significant increase in serum IMA during limb ischemia with sensitivity and specificity over $80 \%$ in cases with clinically severe lower limb involve- ment. In our study we have observed positive correlation between IMA and pain severity measured with the use of VAS. There are very limited data on the correlations between IMA and clinimetric measures of neurological deficits. No correlation between IMA and stroke severity estimated with the use of National Institutes of Health Stroke Scale score (NIHSS) was found in one study [23], but the other [24] reported correlation both with the volume of ischemic lesion evaluated as restriction of diffusion on magnetic resonance imaging (MRI) and with NIHSS score.

According to Falkersammer et al [25] among patients with peripheral arterial occlusive disease (PAOD) IMA was a better predictor of major adverse cardiac events than $\mathrm{N}$-terminal prohormone of brain natriuretic peptide (NT-BNP) or troponin (cTnT).

In diabetic patients oxidative stress and sub-endothelial inflammation resulted in nephropathy, neuropathy and retinopathy. Recently, Chawla et al [26] observed that the higher is elevation of IMA the poorer is glycemic control. Moreover most of the patients with complications showed higher IMA values. In view of the above, it appears that IMA could be related to glycaemic control in type 2 diabetes patients. However, the authors [26] used arbitrary units (ABSU) for the expression of IMA. Thus, the comparison of IMA results between studies requires careful interpretation, which should consider methodological and units 
issues. In our study, we expressed IMA in optical density (O.D.) units and we suggest to call such a biomarker as serum IMA-ABS, when cobalt binding is measured spectrophotometrically, contrary to the units $(U)$ used in enzyme-linked immunosorbent assays (ELISA) based on IMA detection by specific antibodies. Further studies are needed on correlations between results of spectrophotometric methods, which estimate "functional" properties of IMA and ELISA, which measures rather the "mass" of protein.

The serum levels of IMA have been reported to rise in many clinical conditions with acute inflammation, such as sepsis. Yin $\mathrm{M}$ et al. proved that IMA may be treated as predictor of short-term mortality in patients with severe sepsis [27]. It can be also associated with severity of the chronic inflammatory process $[28,29]$.

None of patients included in our study showed clinical symptoms or laboratory markers of acute or chronic inflammation. We can claim that the changes in IMA we have observed were not related to the inflammation.

We observed positive correlation between IMA and homocysteine concentration. Such a relations was not found in the study on polycystic ovary syndrome with or without insulin resistance [30]. However, in type 2 diabetic patients with peripheral arterial disease IMA positively correlated with homocysteine concentration [31]. The results of those studies may suggest that IMA is more related to vascular pathology than to metabolic disturbances.

Moreover, we have noticed positive correlations between IMA and triglycerides and HDL-cholesterol. Moderate correlation between IMA and TAG was already reported, as well as weak correlation with total cholesterol [32]. The relations between IMA and HDL cholesterol we have observed may be caused by higher HDL cholesterol concentration in migraine patients with aura, who have also higher IMA.

IMA formation was also related to the oxidative stress. The correlation between IMA and serum malondialdehyde (MDA), a lipid peroxidation marker, was reported during the course of normal pregnancy [33], thyroid gland dysfunction [34] and obstructive sleep apnea hypopnea syndrome [35].

To conclude, stimulated IMA formation in migraine patients, and particularly in migraineurs with aura, can reflect oxidative stress even during interictal period. It can be independent from acute or chronic inflammation, but in patients with additional hyperhomocysteinemia and/or hypertriacylglycerolemia it could reflect the increased cardiovascular risk.
Acknowledgements

\section{Conflict of interest statement}

The authors declare no conflict of interest.

\section{Funding sources}

There are no sources of funding to declare.

\section{Informed consent and ethical approval}

Informed consent was obtained from all individual participants included in the study. The study design was positively evaluated and approved by the Bioethics Committee at Poznan University of Medical Sciences.

\section{References}

1. Dreier $P$, Reiffurth $C$. The stroke-migraine depolarization continuum. Neuron. 2015 May;86(4):902-922.

2. Leao AAP. Spreading depression of activity in cerebral cortex. J Neurophysiol. 1944;7:359-390.

3. Hadjikhani N, Sanchez Del Rio M, Wu O, Schwartz D, Bakker D, Fischl B et al. Mechanisms of migraine aura revealed by functional MRI in human visual cortex. Proc Natl Acad Sci USA. 2001 Apr;98(8):4687-4692.

4. Fabricius $M$, Akgoren N, Lauritzen M. Arginine-nitric oxide pathway and cerebrovascular regulation in cortical spreading depression. Am J Physiol. 1995 Jul;269(1 $\mathrm{Pt} 2): \mathrm{H} 23-\mathrm{H} 29$.

5. Pezzini A, Del Zotto E, Giossi A, Volonghi I, Grassi M, Padovani A. The migraine-ischemic stroke connection: potential pathogenic mechanisms. Curr Mol Med. 2009 Mar;9(2):215-226.

6. Spector JT, Kahn SR, Jones MR, Jayakumar M, Dalal D, Nazarian S. Migraine headache and ischemic stroke risk: an updated meta-analysis. Am J Med. 2010 Jul;123(7):612-624.

7. Hu X, Zhou Y, Zhao H, Peng C. Migraine and the risk of stroke: an updated meta-analysis of prospective cohort studies. Neurol Sci. 2016 Oct. [Epub ahead of print]

8. Winsvold BS, Sandven I, Hagen K, Linde M, Midthjell K, Zwart JA. Migraine, headache and development of metabolic syndrome: an 11-year follow-up in the Nord-Trøndelag Health Study (HUNT). Pain. 2013 Aug;154(8):13051311.

9. NCEP Expert Panel. Third Report of the National Cholesterol Education Program (NCEP) Expert Panel on Detection, Evaluation, and Treatment of High Blood Cholesterol in Adults (Adult Treatment Panel III) final report. Circulation. 2002 Dec;106:3143-3421.

10. Bigal ME, Kurth T, Santanello N, Buse D, Golden W, Robbins $\mathrm{M}$ et al. Migraine and cardiovascular disease: a population-based study. Neurology. 2010 Feb;23;74(8):628635.

11. Lippi G, Montagnana M, Salvagno GL, Guidi GC. Potential value for new diagnostic markers in the early recognition of acute coronary syndromes. CJEM. 2006 Jan;8:27-31.

12. Bar-Or D, Winkler J, Van Benthuysen $K$, Harris L, Lau E, Hetzel FW. Reduced albumin-cobalt binding with transient myocardial ischemia after elective percutaneous transluminal coronary angioplasty: a preliminary comparison to Creatine kinase-MB, myoglobin and troponin I. Am Heart J. 2001 Jun;141:985-991. 
13. Borderie D, Allanore $Y$, Meune C, Devaux JY, Ekindjian OG, Kahan A. High ischemia-modified albumin concentration reflects oxidative stress but not myocardial involvement in systemic sclerosis. Clin Chem. 2004 Nov;50:2190-2193.

14. harma R, Gaze DC, Pellerin D, Mehta RL, Gregson H, Streather CP. Ischemia-modified albumin predicts mortality in ESRD. Am J Kid Dis. 2006 Mar;47:493-502.

15. Apple FS, Quist HE, Otto AP, Mathews WE, Murakami MM. Release characteristics of cardiac biomarkers and ischemia-modified albumin as measured by the albumin cobalt-binding test after a marathon race. Clin Chem. 2002 Jul;48:1097-1100.

16. El Hasnaoui A, Vray M, Richard A, Nachit-Ouinekh F, Boureau F. MIGSEV Group. Assessing the severity of migraine: development of the MIGSEV scale. Headache. 2003 Jun;43: 628-635.

17. Stewart WF, Lipton RB, Dowson AJ, Sawyer J. Development and testing of the Migraine Disability Assessment (MIDAS) Questionnaire to assess headache-related Disability. Neurology. 2001 Mar;56(Suppl. 1):20-28.

18. Richard A, Henry P, Chazot G, Massiou H, Tison S, Marconnet $R$ et al. Qualité de vie et migraine. Validation du questionnaire QVM en consultation hospitalière et en médecine générale. Therapie. 1993 Mar-Apr;48:89-96.

19. Bar-Or D, Lau E, Winkler JV. A novel assay for cobalt-albumin binding and its potential as a marker for myocardial ischemia-a preliminary report. J Emerg Med. 2000 Nov;19(4):311-315.

20. Bar-Or D, Curtis G, Rao N, Bampos N, Lau E. An insight into the mechanism of a new assay for myocardial ischemia. Eur J Biochem. 2001 Jan;268(1):42-47.

21. Bhagavan NV, Lai EM, Rios PA, Yang J, Ortega-Lopez $A M$, Shinoda $\mathrm{H}$ et al. Evaluation of Human Serum Albumin Cobalt Binding Assay for the Assessment of Myocardial Ischemia and Myocardial Infarction. Clin Chem. 2003 Apr;49(4):581-585.

22. Gunduz A, Mentese A, Turedi S, Karahan SC, Mentese $U$, Eroglu $O$ et al. Serum ischaemia-modified albumin increases in critical lower limb ischaemia. Emerg Med J. 2008 Jun;25(6):351-353.

23. Herisson F, Delaroche O, Auffray-Calvier E, Duport BD, Guillon B. Ischemia-modified albumin and heart fatty acid-binding protein: could early ischemic cardiac biomarkers be used in acute stroke management? J Stroke Cerebrovasc Dis. 2010 Jul-Aug;19(4):279-282.

24. Can S, Akdur O, Yildirim A, Adam G, Cakir DU, Karaman $\mathrm{HI}$. Myelin basic protein and ischemia modified albumin levels in acute ischemic stroke cases. Pak J Med Sci. 2015 Sep-Oct;31(5):1110-1114.

25. Falkensammer J, Frech A, Duschek N, Gasteiger S, Stojakovic $\mathrm{T}$, Scharnagl $\mathrm{H}$ et al. Prognostic relevance of ischemia-modified albumin and NT-proBNP in patients with peripheral arterial occlusive disease. Clin Chim Acta. 2015 Jan;438:255-260.

26. Chawla R, Loomba R, Guru D, Loomba V. Ischemia Modified Albumin (IMA) - A Marker of Glycaemic Control and Vascular Complications in Type 2 Diabetes Mellitus. J Clin Diagn Res. 2016 Mar;10(3):BC13-BC16.
27. Yin M, Liu X, Chen X, Li C, Qin W, Han H et al. Ischemiamodified albumin is a predictor of short-term mortality in patients with severe sepsis. J Crit Care. 2016 Aug;37:7-12.

28. Ozdemir M, Kivici A, Balevi A, Mevlitoğlu I, Peru C. Assessment of ischaemia-modified albumin level in patients with psoriasis. Clin Exp Dermatol. 2012 Aug;37:610-614.

29. Chandrashekar L, Kumarit GR, Rajappa M. 25-hydroxy vitamin $D$ and ischaemia-modified albumin levels in psoriasis and their association with disease severity. $\mathrm{Br}$ J Biomed Sci. 2015 Jul;72(2):56-60.

30. Caglar GS, Oztas E, Karadag D, Pabuccu R, Demirtas $\mathrm{S}$. Ischemia-modified albumin and cardiovascular risk markers in polycystic ovary syndrome with or without insulin resistance. Fertil Steril. 2011 Jan;95(1):310-313.

31. Ma SG, Wei CL, Hong B, Yu WN. Ischemia-modified albumin in type 2 diabetic patients with and without peripheral arterial disease. Clinics (Sao Paulo). 2011;66(10):16771680.

32. Klafke JZ, Porto FG, Batista R, Bochi GV, Moresco RN, da Luz PL et al. Association between hypertriglyceridemia and protein oxidation and proinflammatory markers in normocholesterolemic and hypercholesterolemic individuals. Clin Chim Acta. 2015 Aug;448:50-57.

33. Bahinipati J, Mohapatra PC. Ischemia Modified Albumin as a Marker of Oxidative Stress in Normal Pregnancy. J Clin Diagn Res. 2016 Sep;10(9):BC15-BC17.

34. Seshadri Reddy V, Bukke S, Mahato K, Kumar V, Reddy NV, Munikumar M. A meta-analysis of the association of serum ischemia-modified albumin levels with human hypothyroidism and hyperthyroidism. Biosci Rep. 2016 Dec 5. pii: BSR20160268. [Epub ahead of print]

35. He Y, Chen R, Wang J, Pan W, Sun Y, Han F et al. Neurocognitive impairment is correlated with oxidative stress in patients with moderate-to-severe obstructive sleep apnea hypopnea syndrome. Respir Med. 2016 Nov;120:25-30.

Acceptance for editing: $2016-12-10$ Acceptance for publication: 2016-12-22

Correspondence address: Slawomir Michalak Department of Neurochemistry and Neuropathology Poznan University of Medical Sciences 49 Przybyszewskiego Street, 60-355 Poznan, Poland phone: +48618691443 fax: +48 618691444 email: swami@ump.edu.pl 